\title{
Os herbicidas causam impactos na sobrevivência e desenvolvimento de abelhas?
}

\author{
Herbicides cause impacts on survival and development of bees?
}

\author{
Patricia Andrea Monquero $^{1 *}$, Alessandro Santos Oliveira ${ }^{2}$
}

Resumo - A polinização é um serviço ecossistêmico chave na manutenção da biodiversidade, essencial no processo reprodutivo das plantas superiores em quase todos os sistemas produtivos terrestres. Para que se possa manter a vasta biodiversidade global, incluindo de espécies cultivadas, é necessária que haja diversidade de polinizadores. No entanto, as abelhas sofrem com as ações antrópicas. Estes insetos vêm desaparecendo de áreas agrícolas devido à introdução de espécies exóticas, às grandes áreas de monocultivo, ao desmatamento para agricultura e pastagem e, principalmente, à fragmentação de habitats e ao uso excessivo ou incorreto de defensivos agrícolas. Esses pesticidas podem afetar não só a longevidade das abelhas, como também sua vitalidade o que pode, ao longo do tempo, influenciar na manutenção das colônias. No caso do uso de defensivos agrícolas, a maioria dos estudos visa avaliar os efeitos dos inseticidas sobre as abelhas, sendo escassos os estudos sobre outras classes de produtos, como os herbicidas. Esta revisão buscou dar foco aos possíveis efeitos, especialmente, de uso de herbicidas na sobrevivência e no desenvolvimento das abelhas. O uso de herbicidas que apresentam toxicidade para os insetos benéficos, como glyphosate, 2,4-D, MSMA e paraquat devem ser continuamente monitorado. As doses subletais dos herbicidas, ou seja, dose menor do que a que provoca a morte, podem provocar alterações comportamentais nos indivíduos, acarretando em sérios prejuízos à manutenção das colônias.

Palavras-chave: abelhas sem ferrão, A. mellifera, controle químico, resíduos

Abstract - Pollination is a key ecosystem service in maintaining biodiversity, which is essential in the reproductive process of higher plants in almost all terrestrial production systems. In order to maintain the vast global biodiversity, including cultivated species, there is a need for diversity of pollinators. However, bees suffer from anthropic actions. These insects have been disappearing from agricultural areas due to the introduction of exotic species, large areas of monoculture, deforestation for agriculture and pasture and, mainly, the fragmentation of habitats and the excessive or incorrect use of pesticides. These pesticides can affect not only the longevity of the bees, but also their vitality which may, over time, influence the maintenance of the colonies. In the case of pesticide use, most studies aim to evaluate the effects of insecticides on bees, and studies on other product classes, such as herbicides, are scarce. This review sought to focus on the possible effects, especially, of herbicide use on bee survival and development. The use of herbicides that show toxicity to beneficial insects, such glyphosate, 2,4-D, MSMA and paraquat must be monitored continuously. The sublethal doses of herbicides, that is, lower dose than the one that causes the death, can trigger behaviors in individuals, causing in serious damages to the colonies maintenance.

Keywords: stingless bee, A. mellifera, chemical control, residue

Recebido: Março 08, 2017. Aceito: Dezembro 21, 2017.

${ }^{1}$ Departamento de Recursos Naturais e Proteção Ambiental, Centro de Ciências Agrarias, Universidade Federal de São Carlos - UFSCar, Rodovia Anhanguera, km 173, CP 153, CEP 13600970, Araras, SP, Brasil.

E-mail: pamonque@ufscar.com

${ }^{2}$ Associação dos Meliponicultores - AME-Rio, Rio de Janeiro, RJ, Brasil. E-mail: alsanoliveira@gmail.com 


\section{Introdução}

A polinização é essencial para a reprodução e manutenção da diversidade de espécies de plantas e provê alimentos para humanos e animais, influenciando, também, o aspecto qualitativo da produção (Buchmann e Nabhan, 1996). Na maioria dos ecossistemas mundiais, as abelhas são os principais polinizadores (Biesmeijer e Slaa, 2004).

As abelhas participam da manutenção do fluxo de energia para outras espécies animais, pois fazem parte da base das cadeias tróficas, sendo parte integrante do mecanismo de reprodução vegetal, aumentando a fertilidade e produtividade da maioria de plantas que dependem de polinização cruzada, bem como na manutenção da variabilidade genética da flora (Proní, 2000).

Estima-se que $40 \%$ dos polinizadores existentes sejam abelhas, perfazendo um total de 40.000 espécies diferentes, onde $75 \%$ das culturas e $80 \%$ das espécies de plantas dotadas de flores dependem da polinização animal (Ricketts et al., 2008). Estas espécies são visitantes florais obrigatórios porque dependem desses recursos durante todo o seu ciclo de vida. As larvas das abelhas são alimentadas com mistura de pólen e néctar, e os adultos buscam ativamente o alimento nas flores (Ramalho et al., 1994). O valor da polinização realizado pela abelha Apis mellifera L. 1758 (Hymenoptera, Apidae), é estimado em 5 a 14 bilhões de dólares por ano apenas nos Estados Unidos. Este serviço crítico está agora comprometido pelo declínio da apicultura $(\approx 50 \%$ desde 1950$)$ devido a doenças, perda de subsídios e intoxicação por inseticidas (Kremen et al., 2002).

Além da questão de polinização, vale lembrar que no Brasil, a apicultura forma uma cadeia produtiva composta por mais de 300 mil apicultores e uma centena de unidades de processamento de mel, que juntos empregam, temporária ou permanentemente, quase 500 mil pessoas (IBGE, 2009).

O Brasil contém a maior diversidade de meliponíneos do planeta (Venturieri, 2008). Além das abelhas com ferrão (ex: A. mellifera
L. 1758 (Hymenoptera, Apidae) e A. mellifera scutellata Lepeletier 1836 (Hymenoptera: Apidae: Apinae)), existem mais de 300 espécies de abelhas nativas que contribuem, e muito, para o processo de polinização das culturas e espécies arbóreas. As abelhas sem ferrão (ASF) possuem distribuição geográfica extremamente ampla por todo o país e apresentam grande variabilidade morfofisiológica. As abelhas da subfamília Meliponídea (Hymenoptera, Apidae), são as abelhas indígenas sem ferrão e distribuem-se em duas tribos: a Meliponini, formada apenas pelo gênero Melipona, encontrado, exclusivamente, na região Neotropical (América do Sul, Central e Ilhas do Caribe) e Trigonini, que agrupa um grande número de gêneros e está distribuída mais amplamente no globo terrestre (Kerr et al., 1996). Entre as Trigonini destaca-se a abelha Jataí (Tetragonisca angustula Latreille 1811), a mais comum no Estado de São Paulo e que produz mel de excelente qualidade (Carvalho et al., 2005).

Outras abelhas, pertencentes à família dos Apídeos, são os Bombíneos, as mamangabas, que são extremamente importantes para a polinização de algumas espécies de plantas. Há ainda uma quarta subfamília, os Euglossíneos, compostas pelas abelhas das orquídeas, como a Euglossa cordata L. 1758, que apresentam tamanho relativamente maior (Nogueira Neto, 1997).

Nos últimos anos, a preocupação com a conservação das abelhas tem crescido devido ao declínio dos polinizadores e, consequentemente, da polinização em paisagens densamente ocupadas por atividades econômicas (Allen-Wardell et al., 1998; Kevan e Phillips, 2001). Os fatores que mais contribuem para a redução da diversidade de abelhas são a fragmentação de habitats, que tem sua origem nos desmatamentos; o uso de defensivos agrícolas e a introdução de espécies capazes de competir com as abelhas nativas pelos recursos florais (Allen-Wardell et al., 1998; Kearns et al., 1998).

Os defensivos agrícolas podem afetar diretamente as abelhas causando alterações na dinâmica de 
coleta de recursos e prejudicando a condição da colônia (De La Ru'a et al., 2009). A aplicação de defensivos agrícolas reduz ainda mais a presença de polinizadores em áreas cultivadas, pois os inseticidas utilizados para controlar as pragas podem fazer o mesmo com os insetos polinizadores. Além da problemática relacionada aos inseticidas, os herbicidas quando aplicado promovem o cultivo sem a presença de uma flora heterogênea da comunidade infestante, podendo reduzir a um número mínimo as flores silvestres nas quais as abelhas se alimentam. O resultado final é uma área com uma quantidade muito pequena de polinizadores naturais e uma grande demanda por polinização durante o período de florescimento da densa população de plantas cultivadas (Viana e Silva, 2006).

A partir deste contexto, a presente revisão buscou dar foco aos possíveis efeitos especialmente do uso de herbicidas na sobrevivência e no desenvolvimento das abelhas.

\section{Efeitos de doses subletais de herbicidas nas abelhas}

A maioria dos estudos relaciona a intoxicação das abelhas por defensivos agrícolas a exposição letal, porém a preocupação vem aumentando com os estudos que mostram que níveis subletais, ou seja, dose de uma substância menor do que a que provoca a morte (Boily et al., 2013) podem prejudicar seu comportamento, desenvolvimento e capacidade de combater infecções, causando problemas crônicos provocados pela exposição em longo prazo (Nicolino e Veillerette 2007; Johnson, 2008; Amaro e Godinho, 2012; Boily et al., 2013).

No caso de herbicidas, embora para registro seja necessária a determinação da dose letal - DL50 para abelhas (A. mellifera), existem fatores que precisam ser levados em consideração, como as misturas de tanque (dois ou mais produtos) realizadas pelos agricultores muitas vezes culminando em efeitos que podem ser sinergísticos, inclusive com relação à toxicidade e a resposta diferencial das abelhas nativas a estes produtos. Apenas alguns defensivos agrícolas (abamectina, deltametrina, fipronil, espinosade, malation, paration, metamidofós e tiametoxan) foram testados de modo não padronizado para as abelhas nativas (Nocelli et al., 2012).

Vale ressaltar que, no caso de herbicidas, grande parte são utilizados em pré-emergência ou em pós-emergência inicial, e, portanto, as aplicações são realizadas quando as plantas daninhas ou cultura estão em estado inicial de emergência e desenvolvimento, não sendo atrativas para as abelhas nestes estados fenológicos. $\mathrm{O}$ controle das plantas daninhas deve ser eficiente durante $o$ período crítico de controle, pois nesse momento a competição acarreta em prejuízos a cultura. Depois deste período as plantas daninhas não mais interferem de forma a causar perdas no rendimento da cultura, pois a cultura já fechou a entrelinha, cobrindo o solo (Vargas e Roman, 2006).

Entretanto, é importante considerar que a redução da diversidade floral pode prejudicar o desenvolvimento e a sobrevivência de organismos não alvos. Camilo et al. (2016) verificaram que a ausência de fontes de alimentos como pólen de flores de plantas da família Asteraceae como Bidens pilosa, Emilia fosbergii, Galinsoga parviflora, Sonchus oleraceus e Taraxacum officinale, comprometeu a longevidade e reprodução do parasitoide Palmistichus elaeisis (Delvare, LaSalle, 1993) (Hymenoptera: Eulophidae).

Para entender a influência do herbicida dicamba (ácido benzóico - mimetizador da auxina) sobre os polinizadores, Bohnenblust et al. (2016) aplicaram várias taxas subletais de dicamba nas plantas de Medicago sativa L. (Fabaceae) e Eupatorium perfoliatum L. (Asteraceae) e avaliaram a floração das plantas e a visitação floral por polinizadores. Verificaram que doses de dicamba simulando deriva de partículas $(\approx 1 \%$ da taxa de aplicação em campo) atrasaram o início da floração e reduziram o número de flores de cada espécie vegetal; embora não tenha alterado a qualidade 
do pólen. Além disto, o número de visitas por abelhas foi significativamente menor.

A dose subletal do herbicida metsulfuron-methyl (sulfonilureia - inibidor da enzima acetolactato sintase - ALS) sobre as plantas Mimulus ringens L. (Phyrmaceae), Bidens cernua L. (Asteraceae), Sinapis arvensis L. (Brassicaceae) e Phaseolus vulgaris L. (Fabaceae), reduziu a produção de néctar e a densidade floral em mais de $85 \%$, o que poderia influenciar na visitação de polinizadores (Boutin et al., 2000). Dado que o pólen é a única fonte de proteína para as abelhas, a redução da quantidade e qualidade do pólen (concentração de proteína) devido a exposição a herbicidas pode resultar em consequências negativas para o desenvolvimento e sobrevivência de larvas de abelhas.

Por outro lado, Moffett et al. (1980) verificaram que a pulverização de baixas concentrações de 2,4-D (ácido fenoxiacéticos - mimetizador de auxina) em plantas de algodoeiro não teve efeito significativo sobre os rendimentos da cultura ou na concentração de açúcares do néctar floral e não provocou efeito significativo sobre as visitas das abelhas durante o ano da aplicação.

Entretanto, os problemas mais sérios para as colônias ocorrem quando as abelhas entram em contato direto com os herbicidas devido a problemas de deriva do produto da área agrícola para uma área próxima, como um fragmento florestal ou pelo uso de dessecantes antes da semeadura da cultura, aplicados no florescimento das plantas de cobertura ou das plantas daninhas na área. Muitas plantas daninhas são consideradas atrativas para as abelhas (Tabela 1), sendo fonte de pólen, néctar ou ambos, com presença e florescimento bastante variáveis nas diversas regiões do país (Almeida et al., 2003; Maia-Silva et al., 2012). Existem modelos computacionais que estimam o quanto será a deriva de uma determinada aplicação em um determinado cenário, entretanto, estes modelos foram desenvolvidos em outros países e não levam em consideração algumas condições especificas do Brasil (Cham e Castro Viana Silva, 2017).

A contaminação das abelhas dá-se, em geral, no momento da coleta de néctar e pólen (Jay, 1996), por isso, o tamanho da área pulverizada também influencia os efeitos das substâncias sobre as colônias, pois quanto maior a área, maior a exposição das forrageiras e a contaminação interna da colmeia (Free, 1993). Outro fator a ser considerado é a distância entre as colônias e os campos tratados, cuja relação de exposição aos agrotóxicos é inversamente proporcional a essa distância (Johansen e Mayer, 1990).

Em trabalho publicado por Pettis et al. (2013) a análise de pólen coletado em diferentes áreas dos Estados Unidos, tanto nas culturas, quanto nos estoques das colônias, apresentaram até 35 diferentes ingredientes ativos, sendo encontrados inseticidas e fungicidas em todas as amostras de pólen e herbicidas em $23,6 \%$ das amostras. Os inseticidas esfenvalerato e fosmete estavam em uma concentração maior do que a dose letal mediana em pelo menos uma amostra de pólen. No caso de fungicidas, houve uma correlação direta entre cosumo de pólen contaminado com estes produtos e aumento de infecção das abelhas pelo parasitoide intestinal Nosema cerenae, além de deixar as abelhas mais sensiveis a efeitos toxicos provocados por acaricidas.

Larvas de A. mellifera expostas a diferentes concentrações de paraquat (bipiridílos inibidor do fotossistema I - FSI) no alimento $\left(0,0.001,0.01,0.1\right.$ e $\left.1 \mu \mathrm{g} \mathrm{kg}^{-1}\right)$, apresentaram problemas no seu desenvolvimento embriônico, condensação do citoplasma e formato irregular de células, mesmo com a menor dose utilizada, embora não tenha induzido maior mortalidade (Cousin et al., 2013).

Um estudo recente verificou que o herbicida glyphosate (inibidor da EPSPs) afetou a responsividade gustativa e o desempenho de aprendizagem em abelhas, por meio do teste de extensão de probóscide. No entanto, não foi observado efeito na atividade locomotora quando as abelhas forrageiras 
Tabela 1. Plantas daninhas consideradas atrativas para abelhas.

\begin{tabular}{|c|c|c|c|}
\hline Nome científico & Nome vulgar & Atrativa para abelhas & Família \\
\hline Bidens pilosa & Picão-preto & Sim & Asteraceae \\
\hline Acanthospermum hispidum & Carrapicho de carneiro & Sim & \\
\hline Ageratum conyzoides & Mentrasto & Sim & \\
\hline Emilia sonchifolia & Falsa-serralha & Sim & \\
\hline Galinsoga parviflora & Picão-branco & Sim & \\
\hline Acanthosperum australe & Carrapichinho & Sim & \\
\hline Senecio brasiliensis & Maria-mole & Sim & \\
\hline Sonchus oleraceum & Serralha & Sim & \\
\hline Taraxacum officinale & Dente-de-leão & Sim & \\
\hline Vernonia polyanthes & Assa-peixe & Sim & \\
\hline Vernonia remotiflora & Alumã & Sim & \\
\hline Galinsoga ciliata & Botão-de-ouro & Sim & \\
\hline Baccharis dracunculifolia & Alecrim-do-campo & $\operatorname{Sim}$ & \\
\hline Portulaca oleraceae & Beldroega & Possível & Portulacaceae \\
\hline Euphorbia heterophylla & Amendoim-bravo & Sim & Euphorbiaceae \\
\hline Croton grandulosus & Gervão-branco & Sim & \\
\hline Croton urucurana & Urucurana & Sim & \\
\hline Euphorbia hirta & Erva-de-Santa-Luzia & Possível & \\
\hline Ricinus communis & Mamona & $\operatorname{Sim}$ & \\
\hline Ipomoea asarifolia & Corda-de-viola & Sim & Convolvulaceae \\
\hline Ipomoea bahiensis & Corda-de-viola & Sim & \\
\hline Ipomoea cairica & Corda-de-viola & Sim & \\
\hline Ipomoea hederifolia & Corda-de-viola & Sim & \\
\hline Ipomoea purpurea & Corda-de-viola & Sim & \\
\hline Ipomoea quamoclit & Corda-de-viola & Sim & \\
\hline Merremia aegyptia & Corda-de-viola & Sim & \\
\hline Merremia cissoides & Corda-de-viola & Sim & \\
\hline Raphanus sativus & Rabano & Sim & Brassicaceae \\
\hline Raphanus raphanistrum & Nabiça & Sim & \\
\hline Cajanus cajan & Feijão-guandu & Sim & \\
\hline Lupinus albinus & Tremoço & Sim & \\
\hline Stylosanthes acuminata & Estilosantes & Sim & \\
\hline Stylosanthes grandifolia & Estilosantes & Sim & Fabaceae \\
\hline Stylosanthes guyanensis & Estilosantes & Sim & \\
\hline Stylosanthes scabra & Estilosantes & Sim & \\
\hline Trifolium verticuloso & Trevo & Sim & \\
\hline Crotalaria incana & Guizo-de-cascavel & Sim & Fabaceae - Faboidae \\
\hline Aeschynomene falcata & Angiguinho & Sim (gênero Exomalopsis) & Fabaceae - Faboidae \\
\hline Crotalaria lanceolata & Guizo-de-cascabel & $\operatorname{Sim}$ & Fabaceae - Faboidae \\
\hline Crotalaria anagiroides & Crotalária & Sim & Fabaceae - Faboidae \\
\hline Senna obtusifolia & Fedegoso & Sim & $\begin{array}{c}\text { Fabaceae - } \\
\text { Caesalpinioideae }\end{array}$ \\
\hline Senna ocidentalis & Fedegoso & Sim & $\begin{array}{c}\text { Fabaceae - } \\
\text { Caesalpinioideae }\end{array}$ \\
\hline $\begin{array}{c}\text { Oxalis corniculata } \\
\text { Oxalis latifolia }\end{array}$ & $\begin{array}{l}\text { Trevo } \\
\text { Trevinho }\end{array}$ & $\begin{array}{l}\text { Sim } \\
\text { Sim }\end{array}$ & Oxalidaceae \\
\hline
\end{tabular}

Fonte: Almeida et al. (2003) e Maia-Silva et al. (2012). 
Tabela 1. Continuação...

\begin{tabular}{|c|c|c|c|}
\hline Nome científico & Nome vulgar & Atrativa para abelhas & Família \\
\hline Amaranthus spinosus & Caruru de espinho & Sim & Amaranthaceae \\
\hline Alternanthera tenella & Apaga-fogo & Sim & \\
\hline Apiumlepto phyllum & Aipo bravo & Não & Apiaceae \\
\hline Borreria verticillata & Botão-branco & Sim & \\
\hline Richardia brasiliensis & Poaia-branca & Sim & \\
\hline Spermacoce verticilata & Vassorinha-de-botão & Sim & Rubiaceae \\
\hline Spermacocea latifolia & Erva-quente & Sim & \\
\hline $\begin{array}{c}\text { Cardiospermum } \\
\text { halicacabum }\end{array}$ & Balãozinho & Sim & Sapindaceeae \\
\hline Heliotropium indicum & Crista-de-galo & Sim & Boraginaceae \\
\hline Hyptis densiflora & Cheirosa & Possivel & \\
\hline Hyptis lophantha & Catirina & Sim & Lamiaceae \\
\hline Hyptis suavelens & Cheirosa & Sim & \\
\hline Leonurus sibiricus & Rubim & Sim & \\
\hline Mormodica charantia & Melão-de-são-caetano & Sim & Curcubitaceae \\
\hline Phyllanthus niruri & Espinheira-santa & Possível & Phyllantaceae \\
\hline Phyllanthus tenellus & Quebra-pedra & Possível & \\
\hline Polygala lancifolia & - & Possível & Polygalaceae \\
\hline Polygala paniculata & - & Possível & \\
\hline Waltheria indica & Malva-veludo & Sim & \\
\hline Sida cordifolia & Malva-branca & Sim & \\
\hline Sida glaziovii & Guanxuma-branca & Sim & \\
\hline Sida linifolia & Guanxuma-fina & Sim & \\
\hline Sida santaremnensis & Guanxuma-grande & Sim & Malvaceae \\
\hline Sida spinosa & Guanxuma-de-espinho & Sim & \\
\hline Sida urens & Guanxuma-dourada & Sim & \\
\hline Sida viarum & Guanxuma & Sim & \\
\hline Sinapsis arvenses & Mostarda & Sim & \\
\hline Sida rhombifolia & Guanxuma & Sim & \\
\hline Solanum aculeatissimum & Arrebenta-cavalo & Sim & \\
\hline Solanum agrarium & Arrebenta-boi & Sim & \\
\hline Nicandra physaloides & Joá-de-capote & Sim & Solanaceae \\
\hline Solanum americanum & Maria-pretinha & Sim & \\
\hline Solanum lycocarpum & Lobeira & Sim & \\
\hline Solanum paniculatum & Jurubeba & Sim & \\
\hline Solanum sisymbriifolium & Joá-das-taperas & Sim & \\
\hline Commelina benghalensis & Trapoeraba & Sim & Commelinaceae \\
\hline Nurdaria nudiflora & Trapoeraba & Possível & \\
\hline Avena strigosa & Aveia-preta & Sim & Poaceae \\
\hline Avena sativa & Aveia & Sim & \\
\hline
\end{tabular}

Fonte: Almeida et al. (2003) e Maia-Silva et al. (2012).

coletaram solução de sacarose contaminada com o herbicida em um alimentador artificial, sugerindo que glyphosate pode se acumular dentro da colmeia (Herbert et al., 2014).

Balbuena et al. (2015) testaram o efeito de diferentes concentrações do glyphosate diluidas em uma solução de sacarose sobre A. mellifera. Os pesquisadores verificaram que as abelhas que tinham sido alimentadas com solução contendo $10 \mathrm{mg} \mathrm{L}^{-1}$ de glyphosate passaram mais tempo realizando vôos de regresso do que as abelhas controle (que não receberam esta dieta) ou 
abelhas tratadas com concentrações mais baixas (2,5 $\mathrm{mg} \mathrm{L}^{-1}$ e $\left.5 \mathrm{mg} \mathrm{L}^{-1}\right)$. Além disto, realizaram mais vôos indiretos para a colmeia. Estes resultados sugerem que, nas abelhas, a exposição a níveis de glyphosate comumente encontrados em ambientes agrícolas prejudica as capacidades cognitivas necessárias para recuperar e integrar informações espaciais para um retorno bem-sucedido à colmeia .

Em trabalho realizado com a Bombus morio (Hymenoptera, Apidae) (mamangava), verificou-se que o glyphosate presente no mel e pólen utilizado na alimentação das abelhas, provocou alterações no tecido muscular de voo, com aumento de comprimento das estrias transversais (sarcômeros), assim como no diâmetro das miofibrilas, e estas alterações podem levar ao comprometimento da atividade de polinização nas abelhas expostas ao herbicida, uma vez que altera a atividade de voo (Abdala et al., 2016).

Segundo Freitas e Pinheiro (2010), para um estudo mais realístico do impacto dos defensivos agrícolas, entre eles, os herbicidas sobre os polinizadores, um amplo banco de dados deve ser gerado, na perspectiva de se fornecer subsídios para a análise, interpretação e correlação dos resultados obtidos sob condições de testes de laboratório e de semi-campo e campo às condições dos vários agroecossistemas brasileiros, para a definição de estratégias de manejo visando a otimização do benefício simultâneo dos defensivos agrícolas e polinizadores para as culturas.

Para melhor proteger as abelhas e outros polinizadores, é importante compreender que o risco para as abelhas melíferas poderiam ser minimizadas através da cuidadosa seleção de defensivos agricolas com menor toxicidade e maiores cuidados no momento de aplicação quanto às condições ambientais, evitando deriva e os horários de forregeamento das abelhas no campo.

\section{Efeitos letais de herbicidas}

Os dados publicados são controversos, principalmente devido a ausência de padronização nos trabalhos executados, quanto a forma de exposição e dose testadas. Alguns indicaram que os defensivos agrícolas (herbicidas paraquat e 2,4-D, inseticidas chlorantraniliprole, clothianidin, imidaclopride, thiamethoxam e chlorpyrifos) podem representar um impacto adverso grave (Di Prisco et al., 2013; Larson et al., 2013; Sanchez-Bayo e Goka, 2014), outros trabalhos, por sua vez, indicaram baixo risco ou até mesmo ausência de risco para as abelhas meliferas (2,4-D, paraquat, clothianidin e thiamethoxam) (Cutler e Scott-Dupree, 2007; Pilling et al., 2013; Cutler et al., 2014).

Em campo, observou-se a após a aplicação aérea do herbicida 2,4-D, mortalidade de abelhas adultas e em experimentos controlados a mortalidade foi $20 \%$ maior do que em abelhas que não receberam o herbicida (Almer Jones, 1964). Por outro lado, Moffett e Morton (1971), citados por Que Hee e Sutherland (1981) observaram que as abelhas diretamente pulverizadas com 2,4-D não mostraram nenhuma lesão e nenhum resíduo foi encontrado nas abelhas ou no mel. Estes resultados, no entanto, são questionáveis, já que a DL50 para abelhas meliferas é apenas 1 micrograma / abelha (WSSA, 1994) e em outros estudos com baixas concentrações de 2,4-D, foram verificados redução na produção de crias e mortalidade de abelhas expostas (Johansen e Mayer, 1990; Kidd e James, 1994).

Já os herbicidas paraquat e MSMA (organosenical) foram considerados altamente tóxicos quando colocados no alimento ou usados topicamente nas abelhas (Moffett e Morton, 1973). O paraquat é um potente indutor de stress oxidativo e, a exposição a este herbicida por 12 horas foi altamente letal para Drosophila melanogaster Meigen, 1830 (Diptera, Drosophilidae), com DL50 estimado em $25 \mathrm{mM}$ para machos e $40 \mathrm{mM}$ para fêmeas. Além disto, detectaram aumento dos níveis de peróxido de hidrogênio e resposta na atividade da superóxido dismutase (SOD) que é um antioxidante. Os pesquisadores observaram que sensibilidade ao paraquat foi aumentada durante o acasalamento (Krůček et al., 2015). 
Gonçalves et al. (2016) observaram que os epitélios da glândula salivar e do intestino médio de indivíduos de Podisus nigrispinus Dallas, 1851 (Hemiptera: Pentatomidae) que tiveram contato com o herbicida isoxaflutole (isoxazoles - inibidores da síntese de carotenoides) na dieta apresentaram o núcleo pouco desenvolvido e cromatina condensada, evidenciando baixa atividade metabólica. Observaram também alto grau de apoptose, desorganização e vacúolos secretores no citoplasma epitelial. A porção apical das células do mesêntero mostrou-se pouco desenvolvida, irregular e parcialmente destruída.

Segundo Leite et al. (2016), o herbicida atrazine (triazinas - inibidor do fotossistema II -FSII) reduziu a emergência de fêmeas de Trichogramma bruni Nagaraja 1983 (Hymenoptera, Trichogrammtidae) porém aumentou a de $T$. pretiosum Riley 1879 , T. demoraesi Nagaraja 1983 e T. galloi Zucchi 1988. Além disso, a atrazine reduziu a razão sexual de T. bruni, T. atopovirilia Oatman \& Platner 1983 e T. bennetti Nagaraja \& Nagarkatti 1973, e aumentou a de T. demoraesi. O herbicida foi levemente nocivo para $T$. bennetti e $T$. bruni, mas foi inócuo para as outras espécies de Trichogrammatidae. Além disto, os autores assinalam que as formulações comerciais podem conter muitos sais e adjuvantes que podem ser a causa da mortalidade de organismos não alvos.

Além da intoxicação das abelhas em si, pode ocorrer contaminação dos produtos originados da colméia. Uma avaliação prévia de mel proveniente de Turilândia, MA, indicou a necessidade de monitoramento dos produtos apícolas, uma vez que resíduos de 2,4 D foram detectados (Souza et al., 2013). Em trabalho feito nos Estados Unidos, resíduos de mais de 150 defensivos agricolas (inseticidas, fungicidas e herbicidas) foram detectados em cera, pólen, ou mel (Johnson, 2008).

\section{Conclusões}

O uso de herbicidas que apresentaram toxicidade para os insetos benéficos, como glyphosate, 2,4-D, MSMA e paraquat devem ser continuamente monitorados. Deve ser recomendada a aplicação destes produtos no final da tarde, quando as abelhas campeiras já se recolheram. É importante o desenvolvimento de protocolos de avaliação de riscos e esquemas de mitigação de danos para defensivos agrícolas. Além disto, um sistema de monitoramento dos impactos sobre organismos não alvos deve ser implantado, assim como vem acontecendo em diversos países, para que haja um banco de dados que permita ter uma agricultura de alto rendimento associada a conservação da diversidade biológica.

Além dos efeitos de toxicidade aguda levando as abelhas a morte, doses subletais dos herbicidas podem provocar alterações comportamentais nos indivíduos acarretando em sérios prejuízos a manutenção da colônia.

\section{Referências}

Abdala, F.C.; Costa, M.J.; Sampaio, G.; Camargo, D.A.; Pedrosa, M.; Nogueira, F.L.A. Efeito do cádmio e do glifosato na musculatura de mamangavas. Ciência Tecnologia e Ambiente, v.3, n.1, p.66-72, 2016.

Allen-Wardell, G.; Bernhardt, P.; Bitner, R.; Burquez, A.; Buchmann, S.; Cane, J.; et al. The potential consequences of pollinator declines on the conservation of biodiversity and stability of crop yields. Conservation Biology, v.12, n.1, p.8-17, 1998.

Almeida, D.; Marchini, L.C.; Sodré, G.S.; Ávilla, M.; Arruda, C.M.F. Plantas visitadas por abelhas e polinização. Piracicaba: USP, 2003. Edição Especial. (Série Produtor Rural).

Almer-Jones, T.P. Effect on honey bees of 2,4-D. New Zealand Journal of Agricultural Research, v.7, n.3, p.339-342, 1964.

Amaro, P.; Godinho, J. Pesticidas e abelhas. Revista de Ciências Agrárias, v.35, n.2, p.53-62, 2012.

Balbuena, M.S.; Tison, L.; Hahn, M.L.; Greggers, U.; Menzel, R.; Farina, W.M. Effects of sub-lethal 
doses of glyphosate on honeybee navigation. The Journal of Experimental Biology, v.218, n.17, p.2799-2805, 2015.

Biesmeijer, J.C.; Slaa, E.J. Information flow organization of stingless bee foranging. Apidologie, v.35, n.2, p.143-157, 2004.

Bohnenblust, E.W.; Vaudo, A.D.; Egan, J.F.; Mortensen, D.A.; Tooker, J.F. Effects of the herbicide dicamba on nontarget plants and pollinator visitation. Environmental Toxicology, v.35, n.1, p.144-151, 2016.

Boily, M.; Sarrasin, B.; Deblois, C.; Aras, P.; Chagnon, M. Acetylcholinestarese in honey bees exposed to neonicotinoids, atrazine and glyphosate: laboratory and field experiments. Environmment Science Pollution Research, v.20, n.8, p.5603-5614, 2013.

Boutin, C.; Lee, H.B.; Peart, E.T.; Batchelor, P.S.; Maguire, R.J. Effects of the sulfonylurea herbicide metsulfuron methyl on growth and reproduction of five wetland and terrestrial plant species. Environmental Toxicology and Chemistry, v.19, n.5, p.2532-2541, 2000.

Buchmann, S.E.; Nabhan, G.P. The forgotten pollinators. Washington: Island Press, 1996.

Camilo, S.S.; Soares, M.A.; Leite, G.L.D.; Santos, J.B.; Assis Júnior, S.L.; Zanuncio, J.C. Do floral resources in Eucalyptus plantations affect fitness parameters of the parasitoid Palmistichus elaeisis (Hymenoptera: Eulophidae)? Phytoparasitica, v.44, n.5, p.651-659, 2016.

Carvalho, C.A.L.; Souza, B.A.; Sodré, G.S.; Marchini, L.C.; Alves, R.M.O. Mel de abelha sem ferrão: contribuição para a caracterização físico-química. Cruz das Almas: Universidade Federal da Bahia/SEAGRI-BA, 2005.

Cham, K.O.; Castro Viana Silva, F.E. Nota técnica 02001/6000062/2017-93: avaliação de risco de agrotóxico para insetos polinizadores e lacunas de conhecimento. Brasília: IBAMA, 2017. 18 p.
Cousin, M.; Silva-Zacarin, E.; Kretzschmar, A.; E1 Maataoui, M.; Brunet, J.-L.; Belzunces, L.P. Size changes in honey bee larvae oenocytes induced by exposure to paraquat at very low concentrations. PLoS One, v.8, n.5, p.1-7, 2013.

Cutler, G.C.; Scott-Dupree, C.D. Exposure to clothianidin seed-treated canola has no long-term impact on honey bees. Journal of Economic Entomology, v.100, n.3, p.765-772, 2007.

Cutler, G.C.; Scott-Dupree, C.D.; Sultan, M.; McFarlane, A.D.; Brewer, L. A large-scale field study examining effects of exposure to clothianidin seed-treated canola on honey bee colony health, development, and overwintering success. PeerJ, v.2, n.e652, p.e652, 2014.

De La Rua, P.; Jaffe, R.; Dall'Olio, R.; Munoz, I.; Serrano, J. Biodiversity, conservation and current threats to European honeybees. Apidologie, v.40, n.2, p.263-284, 2009.

Di Prisco, G.; Cavaliere, V.; Annoscia, D.; Varricchio, P.; Caprio, E.; Nazzi, F.; et al. Neonicotinoid clothianidin adversely affects insect immunity and promotes replication of a viral pathogen in honey bees. Proceedings of the National Academy of Sciences of the United States of America, v.110, n.46, p.18466-18471, 2013.

Free, J.B. Insect pollination of crops. London: Academic Press, 1993. 200 p.

Freitas, B.M.; Pinheiro, J.N. Efeitos sub-letais dos pesticidas agrícolas e seus impactos no manejo de polinizadores dos agroecossistemas brasileiros. Oecologia Australis, v. 14, n.2, p.282-298, 2010.

Gonçalves, T.S.; Soares, M.A.; Santos, C.A.; Santos, D.A.; Santos, J.B.; Barroso, G.A. Does the ingestion of isoxaflutole herbicide affect the midgut and salivary glands of pentatomidae predators? Planta Daninha, v.34, n.1, p.125132, 2016.

Herbert, L.T.; Vázquez, D.E.; Arenas, A.; Farina, W.M. Effects of field-realistic doses of glyphosate on honeybee appetitive behaviour. The Journal 
of Experimental Biology, v.217, n.19, p.34573464, 2014.

IBGE - Instituto Brasileiro de Geografia e Estatística. Banco de Dados Agregados. Sistema IBGE de Recuperação Automática - SIDRA. Tabela 74. Brasília: IBGE, 2009. Disponível em: https://sidra.ibge.gov.br/Tabela/74. Acesso em: 21 maio 2009.

Jay, S.C. Spatial management of honey bee on crops. Annual Review of Entomology, v.31, n.1, p.49-65, 1996.

Johansen, C.A.; Mayer, D.F. Pollinator protection: a bee \& pesticide handbook. Cheshire: Wicwas Press, 1990. 212p.

Johnson, R. Recent honey bee colony declines. Washington: Congressional Research Service, 2008. 16 p. Report.

Kearns, C.A.; Inouye, D.W.; Waser, N.M. Endangered mutualism: the conservation of plant pollinator interactions. Annual Review of Ecology and Systematics, v.29, n.1, p.83-112, 1998.

Kerr, W.A.; Carvalho, G.A.; Nascimento, V.A. Abelha uruçu: biologia, manejo e conservação. Belo Horizonte: Acangaú, 1996.

Kevan, P.G.; Phillips, T. The economics of pollinator declines: assessing the consequences. Conservation Ecology, v.5, n.1, p.8-9, 2001.

Kidd, H.; James, D.R. The agrochemicals handbook. 3rd ed. Surrey: Royal Society of Chemistry Information Systems, 1994. 313 p.

Kremen, C.; Williams, N.M.; Thorp, R.W. Crop pollination from native bees at risk from agricultural intensification. Proceedings of the National Academy of Sciences of the United States of America, v.99, n.26, p.16812-16816, 2002.

Krůček, T.; Korandova, M.; Sery, M.; Frydrychova, R.C.; Krůček, T.; Korandova, M.; Szakosova, $\mathrm{K}$. Effect of low doses of herbicide paraquat on antioxidant defense in drosophila. Archives of
Insect Biochemistry and Physiology, v.88, n.4, p.235-248, 2015.

Larson, J.L.; Redmond, C.T.; Potter, D.A. Assessing insecticide hazard to bumble bees foraging on flowering weeds in treated lawns. PLoS One, v.8, n.6, p.e66375, 2013.

Leite, G.L.D.; Paulo, P.D.; Zanuncio, J.C.; Alvarenga, A.C.; Soares, M.A.; Tavares, W.S.; et al. Effects of atrazine-based herbicide on emergence and sex ratio of Trichogrammatidae (Hymenoptera). The Florida Entomologist, v.98, n.3, p.899-902, 2016.

Maia-Silva, C.; Silva, C.I.; Hrncir, M.; Queiroz, R.T.; Imperatriz-Fonseca, V.L. Guia de plantas visitadas por abelhas na caatinga. 1. ed. Fortaleza: Fundação Brasil Cidadão, 2012. 99 p.

Moffett, J.O.; Morton, H.L. Toxicity of airplane applications of 2.4-D, 2,4.5-T. and cotton desiccant to colonies of honey bees. American Bee Journal, v.111, n.2, p.382-383, 1971.

Moffett, J.O.; Morton, H.L. How herbicides affect honey bees. American Bee Journal, v.115, n.5, p.178-179, 1973.

Moffett, J.O.; Stith, L.S.; Morton, H.L.; Shipman, C.W. Effect of 2,4-D on cotton yield, floral nectar, seed germination, and honeybee visits. Crop Science, v.20, n.6, p.747-750, 1980.

Nicolino, F.; Veillerette, F. Pesticides révelations sur un scandale français. Fayard, 2007. 384 p.

Nocelli, R.C.; Roat, T.C.; Zacarin, E.C.S.; Malaspina, O. Riscos de pesticidas sobre as abelhas. In: Semana dos Polinizadores, 3., 2012, Petrolina. Palestras e resumos... Petrolina: Embrapa Semiárido, 2012. (Embrapa Semiárido. Documentos, 249).

Nogueira-Neto, P. Vida e criação de abelhas indígenas sem ferrão. São Paulo: Nogueirapis, 1997. $445 \mathrm{p}$

Pettis, J.S.; Lichtenberg, E.M.; Andree, M.; Stitzinger, J.; Rose, R.; Vanengelsdorp, D. Crop pollination exposes honey bees to pesticides which alters 
their susceptibility to the gut pathogen Nosema ceranae. PLoS One, v.8, n.7, p.e70182, 2013.

Pilling, E.; Campbell, P.; Coulson, M.; Ruddle, N.; Tornier, I. A four-year field program investigating long-term effects of repeated exposure of honey bee colonies to flowering crops treated with thiamethoxam. PLoS One, v.8, n.10, p.e77193, 2013.

Proní, E.A. Biodiversidade de abelhas indigenas sem ferrão na bacia do rio Tabagi, Estado do Paraná, Brasil. Arquivos de Ciência Veterinária e Zoologia, v.3, n.2, p.145-150, 2000.

Que Hee, S.S.; Sutherland, R.G. The phenoxyalkanoic herbicides: chemistry, analysis, and environmental pollution. Boca Raton: CRC Press, 1981. v.1, 319 p.

Ramalho, M.; Giannini, T.C.; Malagodi-Braga, K.S.; Imperatriz-Fonseca, V.L. Pollen harvest by stingless bee foragers (Hymenoptera, Apidae, Meliponinae). Grana, v.33, n.3, p.239-244, 1994.

Ricketts, T.H.; Regetz, J.; Steffan-Dewenter, I.; Cunningham, S.A.; Kremen, C.; Bogdanski, A.; et al. Landscape effect on crop pollinators services: are there general patterns? Ecology Letters, v.11, n.5, p.499-515, 2008.

Sanchez-Bayo, F.; Goka, F. Pesticide residues and bees: a risk assessment. PLoS One, v.9, n.4, p.e94482, 2014.

Souza, J.R.L.; Amarante Junior, O.P.; Brito, N.M.; Franco, C.R.S. Ação de pesticidas sobre abelhas: avaliação do risco de contaminação de méis. Acta Tecnológica, v.8, n.1, p.28-36, 2013.

\section{Vargas, L.; Roman, E. S. Resistência de plantas} daninhas a herbicidas: conceitos, origem e evolução. Passo Fundo: Embrapa Trigo, 2006.

Venturieri, G.C. Criação de abelhas indígenas sem ferrão. 2. ed. rev. atual. Belém: Embrapa Amazônia Oriental, 2008.

Viana, B.F.; Silva, F.O. Limitações e causas do declínio de polinizadores do maracujá amarelo no vale do São Francisco, Juazeiro, BA. In: Encontro sobre Abelhas, 7., 2006, Ribeirão Preto. Anais... Ribeirão Preto, 2006.

WSSA - Weed Society of America. Herbicide handbook. Champaign: WSSA, 1994. 352 p. 